\title{
Safety of Microstimulator During Radiation Therapy - A Preliminary Study on Head and Neck Cancer Patients
}

\author{
Uttam K. Sinha ${ }^{1^{\star}}$, Brenda Villegas ${ }^{1}$, Kuo $C^{2}$, Frances J. Richmond ${ }^{2}$, Rizwan Masood ${ }^{1}$, Nora I. Nelson ${ }^{3}$ and Gerald E. Loeb $^{3}$ \\ ${ }^{1}$ Department of Otolaryngology-Head and Neck Surgery,Keck School of Medicine, USA \\ ${ }^{2}$ Regulatory Science Program, School of Pharmacy, USA \\ ${ }^{3}$ Department of Biomedical Engineering, Viterbi School of Engineering, University of Southern California, Los Angeles, USA
}

*Corresponding author: Uttam K. Sinha, MS, MD, FACS, Medical Director, Head and Neck Surgery, Associate Dean, Surgical Simulation Watt Chair, Head and Neck Cancer Research Keck Medical Center, University of Southern California 1540 Alcazar St, Room 204M, Los Angeles, California 90601, USA, Tel: 323 442.5790; Fax: 323 442.5820; E-mail: sinha@usc.edu

Received date: Jul 22, 2014, Accepted date: Oct 30, 2014, Publication date: Nov 03, 2014

Copyright: ( 2014 Sinha UK, et al. This is an open-access article distributed under the terms of the Creative Commons Attribution License, which permits unrestricted use, distribution, and reproduction in any medium, provided the original author and source are credited.

\begin{abstract}
Objectives: To determine the safety and performance of miniature neuromuscular stimulator called MicroStimulator (MS) during radiation therapy (RT) of head and neck cancer (HNC).

\section{Study Design: Pilot study}

Methods: Four patients who underwent modified radical neck dissection for HNC received MS implants. The implant was placed 2-3 $\mathrm{mm}$ above and parallel to the hypoglossal nerve. All patients had tracheotomy and gastric tubes placed during surgery. Stimulation thresholds were obtained in three patients and electrical exercise programs were initiated in two patients. One patient completed the study, started RT and stimulation of the MS 3-4 weeks after surgery. The implant was energized and controlled by a radio frequency magnetic field from an external coil placed near the implant. Patients underwent stimulation of the MS one hour per day using intermittent trains of stimuli generating fused, maximal contractions.
\end{abstract}

Results: No postoperative or implant specific complication was noted. All patients required and tolerated stimulation without pain or discomfort. Visible contraction of muscles of swallowing was noted in both patients during stimulation indicating that MS remained functional during RT. All patients used the device at home and maintained their oral feeding and weight. None had aspiration pneumonia.

Conclusions: MS appears to be a safe device for stimulation of swallowing muscles during MS for HNC. Implant performance is not affected by RT. It effectively stimulates the nerves to induce robust contraction of muscles of swallowing and laryngeal elevation. High patient compliance was achieved with this implant.

Keywords: Head and neck cancer; MicroStimulator, Radiation therapy; Quality of life; Traditional swallowing therapy

\section{Introduction}

In the pursuit of improving the management of head and neck squamous cell carcinoma (HNC) there has been intensification of radiation therapy (RT) by concurrent delivery of chemotherapy (CT) and altered fractionated RT, notably accelerated regimens [1,2]. These approaches intensify the tumoricidal effects of RT [3]. However, the radiation sensitizing effects of CT lead to increased acute toxicity (e.g. mucositis) and late complications (e.g. fibrosis, stenosis) [4]. Excessive mucositis, fibrosis and reduced motility of the swallowing muscles lead to dysphagia observed following chemoradiation therapy (CRT) [5]. Dysphagia is chronic in nature and has the most detrimental effect on quality of life (QOL). A more serious problem related to dysphagia is silent aspiration, a condition that can cause death. Late pharyngeal toxicity of CRT has recently been recognized as the main barrier to winning the battle with HNC [6]. A prospective cohort study and cross-sectional quality of life (QOL) analysis showed that implementation of pretreatment traditional swallowing therapy (TST) may improve dysphagia specific QOL in HNC patients undergoing CRT $[7,8]$.

A continuous challenge faced by the clinician and patient is compliance with a daily exercise program of TST. We developed a new protocol in our institution for management of dysphagia in patients with HNC (this issue). They undergo TST and transcutaneous neuromuscular electrical stimulation (tNMES) for 1 hour twice a week during CRT. Stimulation via surface electrodes has limitations because of the amount of cutaneous stimulation that patients will tolerate and the need for a qualified caregiver to apply the electrodes and adjust the stimulus parameters. In addition, patient and family spend four to five hours (including commute time) for each therapy session. To improve the efficacy and ease of NMES treatment, we employed an implantable platform technology called MicroStimulator (MS) [9], which can be used to treat neuromuscular disorders effectively at home (Figure 1). 
Page 2 of 5

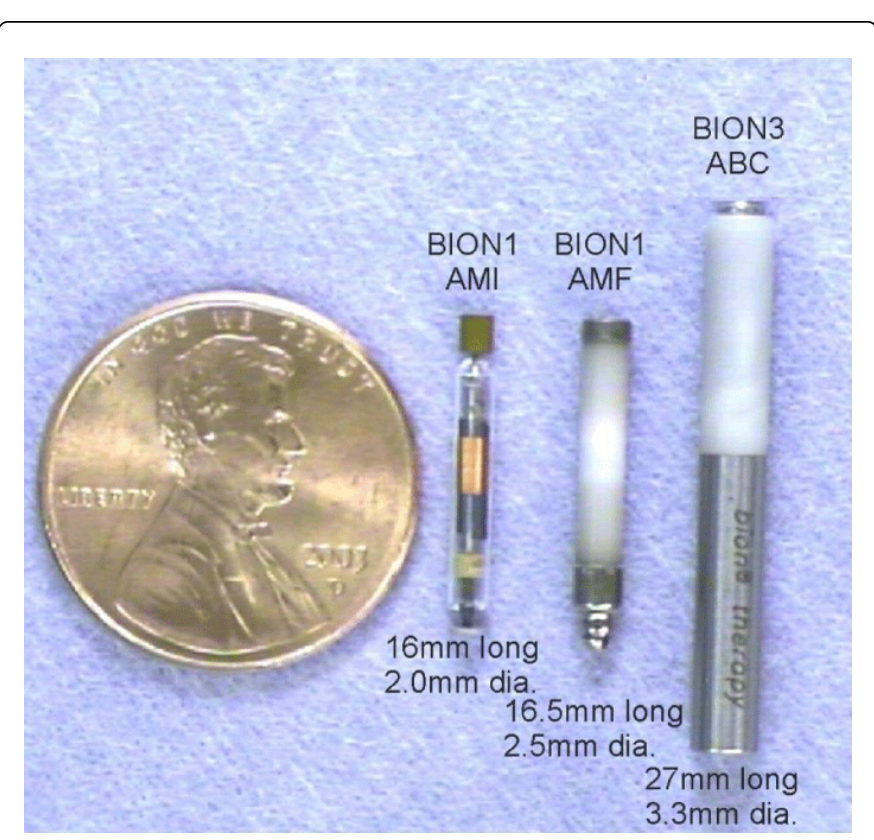

Figure 1: A glass-encapsulated implant powered by a $480 \mathrm{kHz}$ carrier (MS, AMI) was used in this study. The size and shape are compared with a penny and other generations of MS.

A variety of mechanisms are responsible for improvement in swallowing function by NMES therapy. Studies have shown that electrical stimulation increases the local blood flow and reduces the extracellular fluid resulting in decreased edema in the tissues [10]. In addition, NMES selectively activates both type I and type II muscle fibers [11]. The type II fibers are involved in the high-speed forceful contractions of several muscles involved in swallowing [11]. Type II fibers are the first to undergo disuse atrophy but they respond well to electrically induced exercise. Thus therapy directed at type II muscle fibers involved in swallowing should improve swallowing functions in HNC patients with dysphagia. Stimulation of a muscle nerve will elicit centrally propagated activity in large diameter proprioceptive afferents such as from muscle spindles and Golgi tendon organs and antidromic conduction in motor axon collaterals to Renshaw interneurons. Functional magnetic resonance imaging studies of the sensory cortex in stroke patients demonstrate increase in intensity in sensory cortex following similar stimulation of the arm muscles [12,13]. Such ascending activity may facilitate cortical plasticity that helps to maintain or recover voluntary function. Finally, recent data from our laboratory demonstrated that NMES with traditional swallow therapy (TST) reduces fibrosis in the muscles of laryngeal elevation after radiation therapy (RT), and improves dysphagia in HNC patients. RT causes overexpressions of TGF- $\beta 1$, which in turn downregulates the expression of Myo-D gene through Smad3 signaling pathway. Normal expression of Myo-D is important to maintain function of cervical muscles. tNMES with TST restores this signaling pathway, reduces fibrosis and improves dysphagia in HNC patients [14].

The purpose of this study is to establish the safety, to optimize the surgical placement and stimulation parameters, and to determine the performance of the implant during CRT.

\section{Materials and Methods}

\section{Microstimulator}

Microstimulators are a new class of generic wireless devices that can be injected into muscles and near nerves to deliver precisely metered stimulation pulses (Alfred E. Mann Foundation for Scientific Research, Valencia, CA). These injectable, wireless MS receive power and command signals by inductive coupling from an external antenna [15-17]. Four generations of this technology are now either in clinical trials or under development $[9,18,19]$. All are designed to stimulate myelinated sensory or motor axons, typically in peripheral nerves or muscles (Figure 1).

The implants are designed to stimulate myelinated sensory or motor axons, typically in peripheral nerves or muscles. MS has been approved for and used in clinical investigational studies in the U.S., Canada and Italy $[9,18,19]$. The electronic components of the implant are housed in a hermetically sealed glass capsule, $2 \mathrm{~mm}$ diameter x 16 $\mathrm{mm}$ long. Each device delivers monophasic stimulation pulses (0.5$31.5 \mathrm{~mA}, 8-512 \mu \mathrm{s})$ through a tantalum capacitor electrode, which is immediately recharged to the compliance voltage in order to maintain the charge-balance required for safe chronic use in the body. The counter-electrode is made from pure iridium, which forms a conductive, non-polarizing oxide that can be used safely to deliver maximal stimulus pulses [20]. The unpowered implant is an inert foreign body that can be left in place indefinitely when no longer required [21].

MSs are safe in a radiation field. Implantable Technology Testing: Three MSs were implanted in Filet mignon at different orientation as shown in Figure 2 and were exposed to radiation therapy twice with dose of 70 Gy using 137 CS source, gamma cell 40. The MS implants were extracted out from the Filet mignon and tested for their electrical performance and reliability.

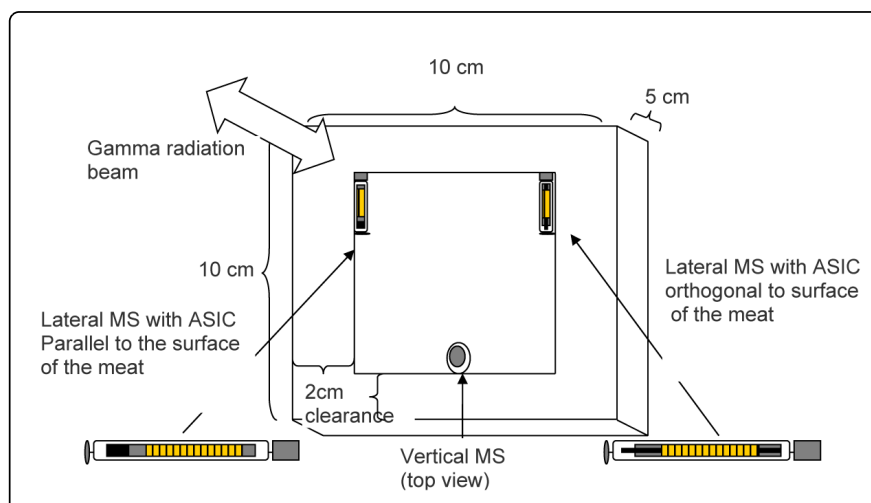

Figure 2: Schematic diagram showed placement of MSs in the beef meat. The implanted MSs were irradiated with $70 \mathrm{~Gy}$ and then tested for their quality performance (as described in materials and methods).

\section{Patient Selection}

The study was performed following approval by the Institutional Review Board and in accordance with Good Clinical Practice under the Investigational Device Exemption from the United States Food and Drug Administration (FDA). All patients underwent a modified 
radical neck dissection (MRND) for HNC. During MRND, the implant was placed 2-3 $\mathrm{mm}$ above and parallel to the hypoglossal nerve and secured in position using absorbable sutures. All patients had a tracheotomy and a gastric tube (G-tube) placed during surgery.
The patient demographics are shown in Table 1. The patients were further assessed through analyzing improvement in tongue mobility and esophageal transfer.

\begin{tabular}{|l|l|l|l|l|l|}
\hline Patient & Age & Site & Stage (TNM) & Surgery & Reconstruction \\
\hline 1 & 63 & BOT & T1N2M0 & PG, MRND & Primary closure \\
\hline 2 & 57 & Mandible & T4NOM0 & SM, MRND & Free flap \\
\hline 3 & 36 & Oral tongue & T1N1M0 & PG, MRND & Primary closure \\
\hline 4 & 67 & Tonsil & T1N2M0 & PP, MRND & Primary Closure \\
\hline
\end{tabular}

Table 1: Patient demographics. BOT: base of tongue, TNM: tumor, node, metastasis, PG: partial Glossectomy, MRND: modified radical neck dissection, SM: segmental mandibulectomy, PP: partial pharyngectomy.

\section{Results}

Before using the MS in the present clinical application, we conducted a series of tests to demonstrate that their electrical performance and reliability would not be affected by exposure to the anticipated gamma radiation treatments of about 70 Gy cumulative exposures. The MS contains a custom integrated circuit chip built using a commercial $0.35 \mu$ CMOS process, which is susceptible to damage from both high rates and high cumulative doses of radiation [22]. The electrical outputs of three MS implants were measured over their full range of parameters (pulse current 0.5-31.5 mA, duration 8-512 $\mu$ s, and frequency 1-200 pps) in a specially instrumented saline bath. They were implanted into a Filet mignon piece of beef with orthogonal orientations so that the beam would pass normal to the plane of the chip in one device and tangential to the plane of the chip along the length and breadth of the chip respectively in the other devices as shown in Figure 2. This phantom was placed in the radiation chamber of a clinical gamma source calibrated to $5485 \mathrm{rad} / \mathrm{h}$ and exposed for 77 minutes to achieve a 70 Gy total dose. The devices were retested for electrical function on the same day and again 10 weeks later. They were then implanted in a fresh piece of meat in the same orientations and subjected to a second 70 Gy irradiation and immediate retest. Thus each implant received 140 Gy radiations. All devices functioned within specification at all test intervals. There was a tendency for the relationship between the commanded and measured output current to decline by up to $20 \%$ immediately after radiation and then to recover over time post irradiation. This is consistent with a small shift in threshold for linear transistors with thick gate oxides due to initial formation and then spontaneous annealing of electron shifts in the semiconductor crystals [23].

During MRND, the MS is implanted above the left hypoglossal nerve and secured in position using sutures in all patients (Figure 3). Only one of the four patients completed the study but stimulation thresholds for visible twitch contractions were obtained in three patients. Patient \#1 was too ill post-operatively to participate and died at home during the fourth post-operative week with acute myocardial infarction. Patient \#2 had a recurrence of the tumor requiring additional surgery but did use the stimulation briefly. Histopathology in patient \#3 demonstrated early stage disease with no lymph node metastases so a decision was made not to irradiate. Patient \#4 started CRT within 3-4 weeks after surgery. Stimulation of the MS implant began before starting CRT. Two sets of stimulation protocols were available depending on the patient's tolerance for muscle contractions: low (low frequency, supramaximal twitch) and high (strong, fused contractions - 10-30 pps). Stimulus strength for both was set at about 3 times threshold to assure complete recruitment of all motor units in the target muscles.

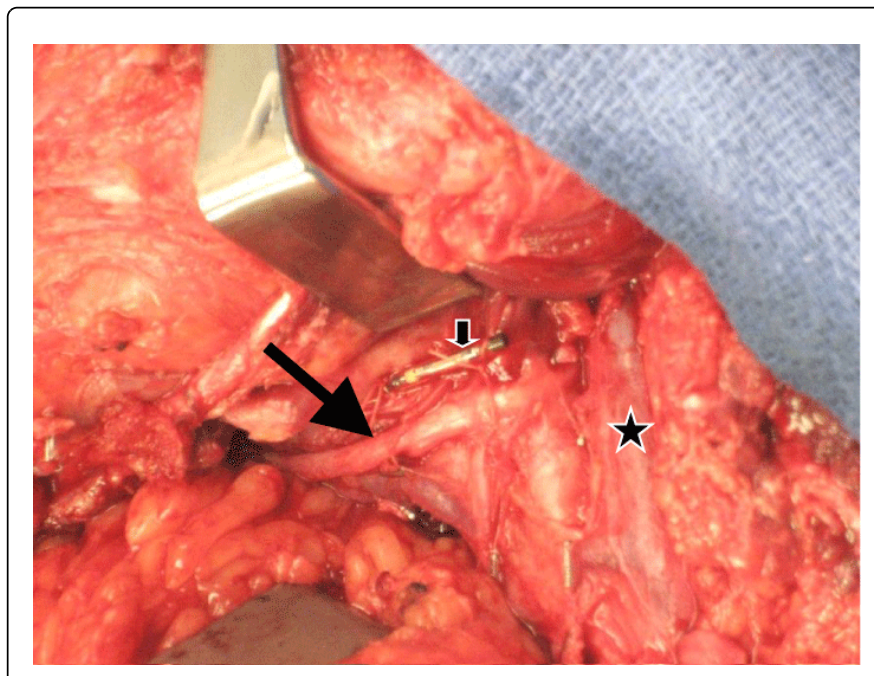

Figure 3: MS (small arrow) is placed above the left hypoglossal nerve (large arrow) during left modified radical neck dissection. Star indicates the origin of ansa cervicalis nerve.

The implant receives both power and digital command signals via an external inductive coil held over the device by a modified soft cervical collar (Figure 4). The command signals specify the address of the implant that should respond to the pulse current $(0.5-31.5 \mathrm{~mA} ; 14$ $\mathrm{V}$ compliance) and duration (8-512 microseconds) for a single output pulse. The coil is connected to a small control box (Personal Trainer) that allows the patient to manually start and stop any of three stimulation programs that are prescribed by the physician or therapist (Figure 4). During out-patient visits, the Personal Trainer is connected to a portable PC running a Windows program that enables testing the implant, monitoring usage information stored in the Personal Trainer, and developing new or modified stimulation programs that can be down-loaded into the Personal Trainer for use at home. Both patients quickly learned and reliably used the prescribed stimulation at home. High stimulation was used for both patients to achieve maximal activation and strong contraction of the muscles of tongue and laryngeal elevation. To prevent fatigue, the system was programmed with an "on and off time" schedule for the strong, intermittent contractions lasting 2-3s every 5-6s. Stimulus strength was ramped up 
Page 4 of 5

and down over $\sim 0.5 \mathrm{~s}$ for comfort. Patients were instructed to apply the stimulation three times daily during the entire course of radiation. Each session lasted for 20 minutes with a total stimulation time of one hour per day. Typically, they stimulated the implant before breakfast, lunch and dinner.

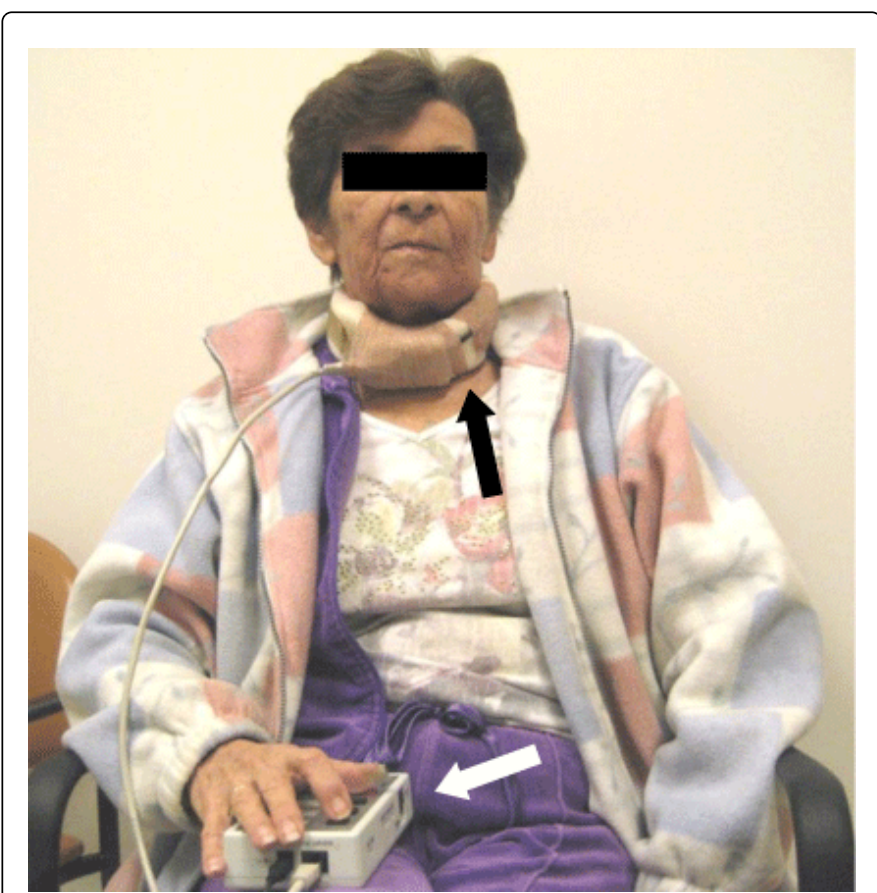

Figure 4: The MS implant receives both power and digital command signals via an external inductive coil held over the device by a modified soft cervical collar (black arrow). The coil is connected to a small control box (Personal Trainer) that allows the patient to manually start and stop stimulation programs (white arrow).

Because the stimulus parameters are adjusted by the clinician on the basis of direct observation of muscle twitches during follow-up visits, there is no need for absolute accuracy in the output current. No postoperative or implant specific complications were noted in the study subjects. Tracheotomy tubes were removed two weeks after surgery. Electrical thresholds for producing visible muscle contractions were in the range 4-6 $\mathrm{mA}$ for $0.2-0.4 \mathrm{~ms}$ pulse durations and were stable over several months of stimulation and follow-up. Both patients who were fitted with exercise programs tolerated the high stimulation well without any pain or discomfort. This pattern of stimulation produced visible, robust contraction of tongue muscles and laryngeal elevation (see video). Patients remained very compliant due to ease of therapy and no commute to the clinic for TST. They maintained their weight and oral feeding with regular diet during CRT. Clinical swallowing evaluations and modified barium swallow (MBS) studies demonstrated that the patients never had penetration or aspiration during the entire course of CRT. None had aspiration pneumonia. After completion of CRT the G-tube was removed. The MS functioned properly during the entire course of RT.

\section{Discussion}

Neuromuscular electrical stimulation (NMES) designed to stimulate peripheral and sensory nerves are gradually becoming a recognized treatment option for neuro-rehabilitation. There is growing scientific evidence of the benefit of NMES. The MS technology platform consists of individual, minimally invasive and low-cost implants that can be used to prevent dysphagia in HNC patients. MS is a new class of generic wireless injectable device which is placed near nerves to deliver precisely metered stimulation pulses. One or more implants receive power and individually addressed command signals by inductive coupling from an external antenna $[15,17]$

One of the concerns regarding electrical stimulation is whether it enhances tumor growth. Epidemiological studies have suggested that long-term exposure to high electromagnetic fields (EMF) may increase the incidence of several cancers [24-26].

Although a variety of biological responses may occur during exposure to exogenous EMF, there is no data that demonstrates increased risk of carcinogenesis under EMF exposure [27]. Shi et al studied this subject extensively and established that when cells were exposed to power line frequency EMF for a prolonged period of time, the critical events of cancer formation did not take place [28]. For the $480 \mathrm{kHz}$ RF frequency used to power the MS implants, almost no energy is absorbed by body tissues. We have used a combined approach of TST and transcutaneous NMES (tNMES) for rehabilitation of dysphagia in HNC patients for the last four years with encouraging results. Survival or disease recurrence has not been affected by tNMES in these patients. However, we encountered a few limitations of tNMES during this study period that prompted us to use MS in HNC patients. Some patients complain of discomfort due to activation of cutaneous afferents during tNMES. The current delivered by the tNMES device provides sub-optimal stimulation of the neuromuscular complex because the motor axons that must be activated are at a substantial distance from the surface electrodes. In addition, patients have to undergo lengthy therapy in the office making this approach more expensive. The MS implant technology has the potential to circumvent the limitations of TST and tNMES. The treatment of dysphagia responds best to daily treatment. Transporting patients daily to clinics and using professional personnel to administer treatment is expensive and quite challenging for the family. The permanently implanted and telemetrically operated MS avoids these problems. This approach has the potential to improve patient compliance and make swallowing rehabilitation more costeffective.

\section{Conclusion}

The present study demonstrated that the MS is a safe implant to use during RT. The performance of the implant is not affected by RT. Patients found it easy to self-administer daily NMES at home and compliance with prescribed treatment was high. MS provides robust contraction of muscles of the tongue and laryngeal elevations without inducing uncomfortable sensations.

\section{Acknowledgement}

The MS devices were built at and provided by the Alfred Mann Institute for Biomedical Engineering at the University of Southern California, which sponsored this clinical trial. 
Citation: $\quad$ Sinha UK, Villegas B, Kuo C, Richmond FJ, Masood R, et al. (2014) Safety of Microstimulator During Radiation Therapy - A Preliminary Study on Head and Neck Cancer Patients. J Nucl Med Radiat Ther 5: 197. doi:10.4172/2155-9619.1000197

Page 5 of 5

\section{References}

1. Fu KK, Pajak TF, Trotti A, Jones CU, Spencer SA, et al. (2000) A radiation oncology group phase III randomized study to compare hyperfractionation and two variants of accelerated fractionation to standard fractionation radiotherapy for head and neck cancer. Int J Radiat Oncol Biol Phys 48: 7-16.

2. Pignon JP, Bourhis J, Domenge C, Designé L (2000) Chemotherapy added to locoregional treatment for head and neck squamous-cell carcinoma: three meta-analyses of updated individual data. MACH-NC Collaborative Group. Meta-Analysis of Chemotherapy on Head and Neck Cancer. Lancet 355: 949-955.

3. Lawrence TS, Blackstock AW, McGinn C (2003) The mechanism of action of radiosensitization of conventional chemotherapeutic agents. Semin Radiat Oncol 13: 13-21.

4. Nguyen NP, Sallah S (2000) Combined chemotherapy and radiation in the treatment of locally advanced head and neck cancers. In Vivo 14: 35-39.

5. Smith RV, Kotz T, Beitler JJ, Wadler S (2000) Long-term swallowing problems after organ preservation therapy with concomitant radiation therapy and intravenous hydroxyurea: initial results. Arch Otolaryngol Head Neck Surg 126: 384-389.

6. Robbins KT (2002) Barriers to winning the battle with head-and-neck cancer. Int J Radiat Oncol Biol Phys 53: 4-5.

7. Carroll WR, Locher JL, Canon CL, Bohannon IA, McColloch NL, et al. (2008) Pretreatment swallowing exercises improve swallow function after chemoradiation. Laryngoscope 118: 39-43.

8. Kulbersh BD, Rosenthal EL, McGrew BM, Duncan RD, McColloch NL, et al. (2006) Pretreatment, preoperative swallowing exercises may improve dysphagia quality of life. Laryngoscope 116: 883-886.

9. Loeb GE, Richmond FJ, Baker LL (2006) The BION devices: injectable interfaces with peripheral nerves and muscles. Neurosurg Focus 20: E2.

10. Breen PP, Corley GJ, O'Keeffe DT, Conway R, Olaighin B (2007) A programmable and portable NMES device for drop foot correction and blood flow assist applications. Med Eng Phys 31: 400.

11. Abdellaoui A1, Préfaut C, Gouzi F, Couillard A, Coisy-Quivy M, et al. (2011) Skeletal muscle effects of electrostimulation after COPD exacerbation: a pilot study. Eur Respir J 38: 781-788.

12. Hara Y, Obayashi S, Tsujiuchi K, Muraoka Y (2013) The effects of electromyography-controlled functional electrical stimulation on upper extremity function and cortical perfusion in stroke patients. Clin Neurophysiol 124: 2008-2015.

13. Golaszewski S, Kremser C, Wagner M, Felber S, Aichner F, et al. (1999) Functional magnetic resonance imaging of the human motor cortex before and after whole hand afferent electrical stimulation. Scand J Rehabil Med 31: 165-167.

14. Peng LP, Masood R, Sinha UK (2008) Radiation therapy in head and neck cancer patients causes muscle fibrosis through increased TGF-B1 downregulating myoD. Proceedings of 7th International Head and Neck Cancer Society: 673.

15. Loeb GE, Zamin CJ, Schulman JH, Troyk PR (1991) Injectable microstimulator for functional electrical stimulation. Med Biol Eng Comput 29: NS13-19.

16. Cameron T, Loeb GE, Peck RA, Schulman JH, Strojnik P, et al. (1997) Micromodular implants to provide electrical stimulation of paralyzed muscles and limbs. IEEE Trans Biomed Eng 44: 781-790.

17. Loeb GE, Peck RA, Moore WH, Hood K (2001) BION system for distributed neural prosthetic interfaces. Med Eng Phys 23: 9-18.

18. Dupont Salter AC, Richmond FJ, Loeb GE (2003) Prevention of muscle disuse atrophy by low-frequency electrical stimulation in rats. IEEE Trans Neural Syst Rehabil Eng 11: 218-226.

19. Weber DJ, Stein RB, Chan KM, Loeb GE, Richmond FJ, et al. (2004) Functional electrical stimulation using microstimulators to correct foot drop: a case study. Can J Physiol Pharmacol 82: 784-792.

20. Brummer SB, Robblee LS, Hambrecht FT (1983) Criteria for selecting electrodes for electrical stimulation: theoretical and practical considerations. Ann N Y Acad Sci 405: 159-171.

21. Cameron T1, Liinamaa TL, Loeb GE, Richmond FJ (1998) Long-term biocompatibility of a miniature stimulator implanted in feline hind limb muscles. IEEE Trans Biomed Eng 45: 1024-1035.

22. Hughes HL, Bendetto JM (2003) "Radiation Effects and Hardening of MOS Technology: Devices and Circuits," IEEE Transactions on Nuclear Science: 50: 500-521.

23. Briere TM, Beddar AS, Gillin MT (2005) "Evaluation of precalibrated implantable MOSFET radiation dosimeters for megavoltage photon beams." Medical Physics 32: 3346-3349.

24. Loomis A, Kromhout H, Kleckner RC, Savitz DA (1998) Effects of the analytical treatment of exposure data on associations of cancer and occupational magnetic field exposure. Am J Ind Med 34: 49-56.

25. Bianchi N, Crosignani P, Rovelli A, Tittarelli A, Carnelli CA, et al. (2000) Overhead electricity power lines and childhood leukemia: a registrybased, case-control study. Tumori 86: 195-198.

26. Caplan LS, Schoenfeld ER, O'Leary ES, Leske MC (2000) Breast cancer and electromagnetic fields--a review. Ann Epidemiol 10:31-44.

27. Sauer H, Stanelle R, Hescheler J, Wartenberg M (2002) The DC electrical-field-induced $\mathrm{Ca} 2+$ response and growth stimulation of multicellular tumor spheroids are mediated by ATP release and purinergic receptor stimulation. J Cell Sci 115: 3265-3273.

28. Shi B, Farboud B, Nuccitelli R, Isseroff RR (2003) Power-line frequency electromagnetic fields do not induce changes in phosphorylation, localization, or expression of the 27-kilodalton heat shock protein in human keratinocytes. Environ Health Perspect 111: 281-287.
This article was originally published in a special issue, entitled: "Cancer Radiation Therapy", Edited by University of Arkansas for Medical Sciences, USA 